\title{
Velocity Distributions \& Density Fluctuations in a 2D Granular Gas
}

\author{
J. S. Olafsent and J. S. Urbach \\ Department of Physics, Georgetown University, Washington, D.C. 20057
}

(November 20, 2018)

\begin{abstract}
Velocity distributions in a vibrated granular monolayer are investigated experimentally. Non-Gaussian velocity distributions are observed at low vibration amplitudes but cross over smoothly to Gaussian distributions as the amplitude is increased. Cross-correlations between fluctuations in density and temperature are present only when the velocity distributions are strongly non-Gaussian. Confining the expansion of the granular layer results in nonGaussian velocity distributions that persist to high vibration amplitudes.
\end{abstract}

PACS numbers: 81.05 Rm, 5.20.D, 05.70.Ln, 83.10.Pp

Typeset using REVTEX 
The effects of inelasticity on the statistical properties of a granular gas has been a topic of recent intense theoretical and experimental interest. In freely cooling granular media, analytic results and simulations show that dissipative inter-particle collisions result in clustering [1], non-Gaussian velocity distributions [2], and eventually to the breakdown of hydrodynamics [3]. In driven granular gases, where the energy lost through collisions is balanced by energy input from external forcing, the effects of inelasticity can be observed in the steady state statistical properties of the gas. Experimental studies have shown clustering [4,5] and non-Gaussian velocity distributions [4,6, [], but the dynamical origin of the velocity distributions remains unclear. A model of a granular gas coupled to a thermal reservoir shows long-range correlations in density and velocity, and velocity distributions that fall off with $v^{3 / 2}$ in the tails [8]. A model with a discrete random forcing produces strong clustering, and a cross-correlation between the fluctuations in density and granular temperature (average kinetic energy) [9]. The cross-correlation is due to the same mechanism as the clustering instability in a freely cooling granular gas: fluctuations of increased density result in more frequent inter-particle collisions, producing increased dissipation and a reduced local granular temperature.

Recent work has demonstrated various collective phenomena for a large number $(\approx$ 20000) of identical, uniform ball bearings constituting less than one layer coverage on a vertically shaken, horizontal plate [4,10]. At peak plate accelerations above $1 \mathrm{~g}$ and for most densities, the particles behave as a rapidly fluctuating gas. Reducing the acceleration amplitude decreases the mean square velocity, or granular temperature, $\mathrm{T}_{G}=<v^{2}>/ 2$, and effectively "cools" the gas, leading to an observed increase in clustering. Continued cooling eventually leads to the formation of a collapse, a condensate of motionless particles that remain in contact with the plate and each other. In the range of $0.8-1.0 \mathrm{~g}$, all of the velocity distributions of the gas appear to scale with the second moment of the distribution to an universal curve [4]. The velocity distributions demonstrate strong deviations from a Gaussian distribution in both the high velocity tails and at low velocities.

This report presents the results of a further investigation into the nature of the non- 
Gaussian velocity distributions and their relation to the observed density fluctuations that stem from the inelastic collisions. In order to understand the non-Gaussian velocity distributions seen previously in this system [4], measurements were made over a larger range of $\Gamma=A(2 \pi \nu)^{2} / g$, the dimensionless acceleration of the plate, where $\nu$ is the frequency in $\mathrm{Hz}$ and $\mathrm{g}$ is the acceleration due to gravity. Our results demonstrate both non-Gaussian velocity distributions and a cross-correlation between density and temperature, similar to that seen in the model system of Puglisi et al. [9]. However, the data reported here clearly shows that the two effects are independent: there is a large range over which the velocity distributions are non-Gaussian but for which the granular temperature is not measurably dependent on the density. Only at low $\Gamma$, where the clustering is the strongest, is the cross-correlation between density and temperature observed in our experiment.

For these experiments, the plate vibration is sinusoidal, $\nu=70 \mathrm{~Hz}$ and unless otherwise stated, the particle density is $\rho=0.532$. For accelerations below $\Gamma=1.25$, the system was initially fluidized by shaking at $\Gamma=1.25$. Two different particle species were used: The small spheres were 302 stainless steel with an average diameter of $0.1191 \mathrm{~cm} \pm 0.00024 \mathrm{~cm}$ and the large spheres were 316 stainless with an average diameter of $0.1588 \mathrm{~cm} \pm 0.00032$ cm. The coefficient of restitution for both particle species is approximately 0.9 [4].

The measured velocity distributions at $\Gamma=0.93, \Gamma=1.50$ and $\Gamma=3.00$ are shown in Fig. 1. The distribution crosses over from one with approximately exponential tails as reported in [4] to a Gaussian distribution. This remarkable evolution is superficially similar to what is observed in freely cooling granular media, where an initial Gaussian velocity distribution becomes non-Gaussian as the system cools. In that case, the evolution is determined by the strength of the inelasticity and the integrated number of collisions per particle, which does not have an obvious analog in the driven system.

As previously reported, the non-Gaussian velocity distributions observed at low accelerations are accompanied by clustering, as demonstrated by a dramatic increase in the structure of the pair correlation function. Figure 2(a) shows the pair correlation function determined via analysis of images taken from above the granular layer for the same accelerations as the 
velocity distributions in Fig. 1. The results of the measured correlation function approach that of an uncorrelated dilute hard sphere gas (solid line). Thus, the crossover to Gaussian velocity distributions is accompanied by the disappearance of spatial correlations, consistent with the suggestions that the non-Gaussian velocity distributions arise from a coupling between density and temperature fluctuations [9].

Increasing the steady state kinetic energy of the granular gas by increasing the amplitude of the acceleration at constant frequency causes the gas to change from primarily twodimensional, where the particles never hop over one another, to essentially three-dimensional [11. This transition can be observed in the pair correlation function, $G(r)$, by the increase in its value for $r<1$. (The correlation function includes only particle separations in the hortizontal plane.) This transition can affect the dynamics in several ways: the effective density is decreased, so that excluded volume effects are less important; the inter-particle collisions can occur at angles closer to vertical, affecting transfer of energy and momentum from the vertical direction to the horizontal; and the change in the dimensionality itself can have important consequences. In order to separate these effects from the direct consequences of increasing the kinetic energy of the gas, a plexiglass lid was added to the system at a height of $0.254 \mathrm{~cm}$, or 1.6 ball diameters for the larger particles. For this plate-to-lid separation, the larger particles cannot pass over top of one another, although enough room remains for collisions between particles at sufficiently different heights to transfer momentum from the vertical to the horizontal direction.

Figure 2 (b) demonstrates the persistence of the particle-particle correlations when the system is constrained to 2D. The particle-particle correlation function decreases slightly from $\Gamma=0.93$ to $\Gamma=1.50$, and then remains essentially constant up to $\Gamma=3$. The small value of $G(r)$ for distances less than one ball diameter indicates that the system remains $2 \mathrm{D}$ as $\Gamma$ is increased. The structure observed in the correlation function is essentially the same as that of an equilibrium elastic hard sphere gas at the same density, indicating that the correlations that exist are due to excluded volume effects.

Figure 3(a) shows that the presence of the lid adds an energy sink to the system at high 
$\Gamma$. At low acceleration $(\Gamma \leq 1)$, very few, if any, particles strike the lid and the lid has no significant effect. At larger $\Gamma$ it is clear that the horizontal granular temperature is reduced as a significant number of particles strike the lid, dissipating energy. It is interesting to note that $\mathrm{T}_{G}$ approaches zero lineraly at finite $\Gamma$, indicating that the relationship between the driving and the horizontal granular temperature is of the form $\mathrm{T}_{G} \propto \Gamma-\Gamma_{c}$.

In order to demonstrate the effect of the lid on the velocity distributions, we use a simple quantitative measure of the non-Gaussian nature, the flatness (or kurtosis) of the distribution:

$$
F=\frac{<v^{4}>}{<v^{2}>^{2}} .
$$

For a Gaussian distribution, the flatness is 3 and for the broader exponential distribution, the flatness is 6 . In the absence of a lid, the flatness demonstrates a smooth transition from non-Gaussian to Gaussian behavior as the granular temperature is raised (Fig. (3)(b)) whether the smaller (circles) or larger (stars) particles are used. With the lid on, the velocity distributions remain more non-Gaussian than in the free system for identical granular temperatures and density (diamonds). The crossover from Gaussian to non-Gaussian behavior observed without the lid is therefore not simply an effect of increasing the vertical kinetic energy of the particles, but rather related to the transfer of energy from the vertical to horizontal motion in the system via collisions, the change in the density, or the change in dimensionality of the gas.

To determine the relative contribution of density changes to the non-Gaussian velocity distributions in the gas, the number of particles on the plate was increased by $15 \%$ and decreased by $10 \%$ from the value of $\rho=0.532$ and the lid was kept on. For all accelerations, the flatness decreased with increased density. This surprising result may be related to the fact that strongly non-Gaussian distributions observed at low $\Gamma$ are accompanied by strong clustering [4]. If the average density is increased, the larger excluded volume means that less phase space remains for fluctuations to persist. The fact that increasing the density with the lid on makes the velocity distribution more Gaussian suggests that the crossover 
to Gaussian observed without the lid is not due to the decrease in density of the gas.

Puglisi et al. 90 have proposed a model which relates strong clustering to non-Gaussian velocity distributions in a driven granular medium. In their framework, at each local density the velocity distributions are Gaussian, and the non-Gaussian behavior arises from the relative weighting of the temperature by local density in the following manner:

$$
P(v)=\sum_{\text {boxes }} n(N) e^{-\left(\frac{v^{2}}{v_{0}^{2}(N)}\right)}
$$

where $v_{0}^{2}(N)$ is the second moment of the distribution for the number of boxes, $n$, that contain $\mathrm{N}$ particles. In this model, the local temperature is a decreasing function of the local density, and the velocity distributions conditioned on the local density are Gaussian.

In our experiment, this feature can be examined by conditioning the local horizontal granular temperature on the local density. That is, examining the distribution of velocities for data at a constant number of particles in the frame of the camera in the strongly clustering regime at $\Gamma \approx 0.8[4]$. In the strongly clustering regime, we do observe a direct correlation between local density and temperature. Figure 4 is a plot of the local temperature as a function of particle number in the camera frame normalized by the granular temperature, including data from the open system for both the smaller and larger particles as well as for the confined system using the larger particles.

The result is similar to the model of Puglisi et al. [9]: At low $\mathrm{T}_{G}$, when the particleparticle correlations are strongest (and larger than those of an equilibrium hard sphere gas [4]), there is a density dependence to the granular temperature (filled circles). At $\Gamma=3$, where all of the particles are essentially uncorrelated in a 3D volume in the absence of a lid, there is no density dependence (open circles, stars). However, even in the confined system at $\Gamma=3$, where the distribution is still not Gaussian, no appreciable density dependence is observed (diamonds), suggesting that the non-Gaussian velocity distributions and densitydependent temperature are not as simply dependent upon one another as they are in the model of Puglisi et al. [9]. In fact, while there is a clear density dependence on the local temperature at low $\Gamma$, the measured velocity distribution conditioned on the local tempera- 
ture is not Gaussian. At each density, the velocity distribution function is almost identical to that of the whole: when the entire distribution is non-Gaussian, the distribution at a single density is non-Gaussian, and when the whole is Gaussian, each conditional velocity distribution is Gaussian.

A more general form of Eq. 2 represents the total velocity distribution as a product of local Gaussian velocity distributions with a distribution of local temperatures:

$$
P(v)=\int_{\vec{r}(t)} f(T(\vec{r}(t))) e^{-\left(\frac{v^{2}}{T(\vec{r}(t))}\right)} d \vec{r} d t
$$

where $T(\vec{r}(t))$ is the local temperature that is varying in space and time. Conditioning on the local temperature would then recover the underlying Maxwell statistics in the fluctuations [12].

Performing this analysis on our data does not succeed in producing Gaussian statistics. Within small windows of local temperature, the distributions remain non-Gaussian. Indeed, the analysis can be extended to condition on both the local temperature and density in the system, but with similarly limited success except for the slowest of particles in the most dilute regions of the system, although all of the conditioned distributions are closer to Gaussian than the full distribution. Curiously, if the local velocities are normalized by the magnitude of the local granular temperature, then the total distribution is Gaussian [12].

These results demonstrate several important new characteristics of a $2 \mathrm{D}$ granular gas. Extreme non-Gaussian velocity distributions observed previously [4] involve strong crosscorrelations between density and velocity fluctuations. This is not the whole picture, however, as a 2D constrained granular gas also demonstrates strongly non-Gaussian behavior without a significant cross-correlation between density and velocity distributions. Finally, the crossover to Gaussian distributions observed when the gas is fully 3D is probably associated with the change in dimensionality or momentum transfer, rather than the increase in kinetic energy or the decrease in density.

This work was supported by an award from the Research Corporation, a grant from the Petroleum Research Fund and grant DMR-9875529 from the NSF. One of us (JSU) was 
supported by a fellowship from the Sloan Foundation. 


\section{REFERENCES}

$\dagger \quad$ email: olafsen@physics.georgetown.edu

[1] S. McNamara and W. R. Young, Phys. Rev. E 53, 5089 (1996).

[2] S. E. Esipov and Th. Pöschel, J. Stat. Phys. 86, 1385 (1997).

[3] I. Goldhirsch and G. Zanetti, Phys. Rev. Lett. 70, 1619 (1993).

[4] J. S. Olafsen and J. S. Urbach, Phys. Rev. Lett. 81, 4369 (1998).

[5] A. Kudrolli, M. Wolpert, and J. P. Gollub, Phys. Rev. Lett. 78, 1383 (1997).

[6] W. Losert, D. G. W. Cooper, J. Delour, A. Kudrolli, and J. P. Gollub, to appear in Chaos.

[7] S. Warr, G. T. H. Jacques, and J. M. Huntley, Powder Tech. 81, 41 (1994); S. Warr, J. M. Huntley, and G. T. H. Jacques, Phys. Rev. E 52, 5583 (1995).

[8] T. P. C. van Noije and M. H. Ernst, Granular Matter 1, 57 (1998).

[9] A. Puglisi, V. Loreto, U. M. B. Marconi, A. Petri, and A. Vulpiani, Phys. Rev. Lett. 81, 3848 (1998).

[10] W. Losert, D. G. W. Cooper, and J. P. Gollub, Phys. Rev. E 59, 5855 (1999).

[11] Visit www.physics.georgetown.edu/ granular to view the actual high speed dynamics.

[12] C. Bizon, M. D. Shattuck, J. B. Swift, and H. L. Swinney, /cond-mat 9904132. 


\section{FIGURES}

FIG. 1. Log-linear plot of velocity distribution functions for increasing $\Gamma$ at constant frequency. As the acceleration is increased, the distributions go from having nearly exponential to Gaussian tails. $(\bigcirc) \Gamma=0.93,(+) \Gamma=1.5,(\square) \Gamma=3.0$.

FIG. 2. (a) Pair correlation functions for the velocity distributions in Figure 1 ( $\bigcirc) \Gamma=0.93$, $(+) \Gamma=1.5,(\triangle) \Gamma=2.0,(\square) \Gamma=3.0$. (b) Pair correlation functions for the velocity distributions where a lid constrains the system to remain 2D. The particle correlations remain as the shaking amplitude is increased.

FIG. 3. (a) Plot of the horizontal granular temperature, $\mathrm{T}_{G}$, as a function of dimensionless acceleration, $\Gamma$, with and without a lid. Data for $\rho=0.532:(\bigcirc) \mathrm{d}=1.2 \mathrm{~mm}$ and $(*) \mathrm{d}=1.6 \mathrm{~mm}$ without lid; $(\diamond) \mathrm{d}=1.6 \mathrm{~mm}$ with lid. Data for $\rho=0.478(\triangle)$ and $\rho=0.611(\square)$ with a lid and $\mathrm{d}=1.6 \mathrm{~mm}$. (b) Plot of the flatness as a function of granular temperature with and without a lid in the system. At low $\Gamma$ the system is nearly $2 \mathrm{D}$ and the lid has no effect.

FIG. 4. Plot of $T(N) / T_{G}$ where $\mathrm{N}$ is the number of particles in the camera frame (see text). At low $\Gamma$, the local temperature and density are strongly correlated. At high $\Gamma$, the local granular temperature is independent of density, even when the velocity distributions remain non-Gaussian in the constrained system. 